\title{
BIOINFORMATICS: INFLAMMATORY CYTOKINES AND ATTENUATION OF DIABETES HYPERCHOLESTEROLEMIA-INDUCED RENAL INJURY USING MORNING GLORY AND NECKLACE POD EXTRACTS
}

\author{
HANAN F ALY ${ }^{1}$, HOWAIDA I ABD-ALLA ${ }^{2 *}$, SANAA A ALI ${ }^{1}$, REDA ABA ALEZ ${ }^{3}$, MOHAMMED T ABU-KRISHA ${ }^{3}$, \\ MOHAMED M MAMDOUH ${ }^{3}$
}

\begin{abstract}
${ }^{1}$ Department of Therapeutic Chemistry, National Research Centre, Dokki, Giza, Egypt. ${ }^{2}$ Department of Chemistry of Natural Compounds, National Research Centre, Dokki, Giza, Egypt. ${ }^{3}$ Department of Information Systems and Computer Engineering, Faculty of Engineering, Al-Azhar University, Cairo, Egypt. Email: howaida_nrc@yahoo.com
\end{abstract}

Received: 25 May 2017, Revised and Accepted: 09 August 2017

ABSTRACT

Objective: The present research in bioinformatics focuses on pharmacological effects of morning glory and necklace pod ethanolic extracts (MGE and NPE) on some biochemical parameters in high fat diet-induced hypercholesterolemia and streptozotocin-induced hyperglycemia in rats.

Methods: Compared to atorvastatin; an anti-hypercholesterolemic (HC) and glibenclamide; an antidiabetic drug. Endothelium activation markers of soluble vascular cell adhesion molecule (VCAM)-1 and intercellular adhesion molecule (ICAM)-1 were determined using enzyme-linked immunosorbent assay. Creatinine, urea, and inflammatory biomarkers; C-reactive protein (CRP) and pro-inflammatory cytokines including tumor necrosis factor alpha (TNF- $\alpha$ ) and interleukin (IL)-10 levels were also measured in serum of different therapeutic groups.

Results: Significant decrease in ICAM-1 level with MGE and NPE supplemented to normal rats as compared to untreated control with percentages decrease 17.80 and $12.00 \%$ was observed. Insignificant change was detected in VCAM-1 level. Profound amelioration in CRP, total urea and creatinine levels by NPE treatment. Creatinine, urea, CRP, and TNF- $\alpha$ level were significantly increased in hyperglycemic (HG)-HC rats. However, IL-10 level showed a significant decrease. Meanwhile, histopathological investigation of the kidney and heart was carried out. Image recognition system for kidney and heart images was developed to diagnose their diseases. Tested extract attenuated creatinine, urea, CRP, and TNF- $\alpha$ level. Hyperglycemia and hypercholesterolemia linked kidney disorders were relieved.

Conclusion: In vivo oral administration with each extract declared suppression of cytokines mediated inflammation, vascular function leading to infiltration reduction of renal macrophage together with lowering in kidney indices and ameliorate renal tissues architectures in HG-HC rats.

Keywords: Morning glory, Necklace pod, Inflammatory, Cell adhesion, Kidney function, Statistic, Image recognition.

(C) 2017 The Authors. Published by Innovare Academic Sciences Pvt Ltd. This is an open access article under the CC BY license (http://creativecommons. org/licenses/by/4. 0/) DOI: http://dx.doi.org/10.22159/ajpcr.2017.v10i11.20119

\section{INTRODUCTION}

Chronic inflammation leading to immune system activation is deeply involved in the pathogenesis of diabetes [1]. It was reported that diabetic atherosclerosis is a disease of hypercholesterolemia associated with an inflammatory status involving several mediators as C-reactive protein (CRP), cytokines, tumor necrosis factor alpha (TNF- $\alpha$ ), and interleukin (IL)-10 [2]. It was found that the treatment of monocytes with a high concentration of glucose leads to the release of several inflammatory cytokines, chemokines, and mediators. Many of them are regulated by the proinflammatory cytokine and nuclear factorkappa $\beta$ (NF- $\kappa \beta$ ) [3]. The activation of inflammatory cytokines, TNF- $\alpha$ and CRP in Type 2 diabetes may be relying on cytokines that inhibit insulin signaling, and glucose transport protein (GLUT)-4 [1], while IL10 is involved in $\beta$-cell hyperactivity [4]. It was found that inflammation and the impairment of vascular function stimulated by risk factors such as hypercholesterolemia, hypertension, and smoking or diabetes are closely related. Irrespective of its cause, inflammation connected with cardiomyopathy, although, there are debatable findings referring inflammation-related atherosclerosis [5]. The choice biomarkersrelated inflammation is found to be connected with various infective procedures in atherogenesis; vascular cell adhesion molecule (VCAM)-1, intercellular adhesion molecule (ICAM)-1, and anti-inflammatory IL-10.

Medicinal plant extracts often consist of complex mixtures of active primary and secondary metabolites and their uses in medicine, against inflammation, and kidney diseases are still based on knowledge from traditional medicinal practice $[4,5]$.

The genera of Sophora and Ipomoea are a prolific source of structurally diverse bioactive metabolites with interesting activities [6,7]. The chemical review on necklace pod ([NP], Sophora tomentosa, reveals the presence of alkaloids, polysaccharides, isoprenylated flavonoids, isoflavonones, flavones, flavonols, and their glycosides. Such compounds have been reported to have diverse bioactivities including antioxidant and immunoregulatory [6,8]. It was reported that the extracts of S. tonkinensis and Sophora flavescens significantly control the levels of insulin, through stimulation of glucose transporter 4 (GLUT-4) which is attenuated by AMP-activated protein kinase (AMPK) pathway while being non-toxic for mice [9].

Many phytochemical studies [7], declared that Ipomoea contains many bioactive secondary metabolites, such as phenolic acids, flavonoids, organic acids, and anthocyanins, with promising effects in ameliorating different disorders such as antinociceptive, antioxidative, and antiinflammatory effects. The isolated anthocyanins decreased the atherosclerotic progress, ameliorated oxidative stress and endothelial dysfunction in mice [10].

To the last of our knowledge, the attenuation of diabetes hypercholesterolemia-induced renal injury by the extracts of morning glory (MG) nor NP have never been discussed before. In vivo, this research 
aims to statistically investigate the action of each extract on some biochemical parameters in high fat diet-induced hypercholesterolemia, and streptozotocin (STZ)-induced hyperglycemia in rats compared to atorvastatin; an anti-hypercholesterolemic (HC) and glibenclamide; an antidiabetic drug.

In bioinformatics [11,12], a new developed image recognition system was developed to investigate renal and heart injuries images. Statistical system for extracting statistical information from the blood tests data of the rats was illustrated in another article [3]

\section{MATERIALS AND METHODS}

\section{Plant materials}

Aerial parts of the MG (Ipomoea tricolor Cav., Family: Convolvulaceae) and the NP (S. tomentosa L., Family: Fabaceae) (Leguminosae) were collected in May 2014 from El Qanater, Qalyubia Governorate. The identification of the plants material was performed by Treas Labib, consultant of plant taxonomy at the Ministry of Agriculture and exdirector of Orman Garden, Giza, Egypt. A voucher sample is kept in the herbarium of National Research Centre (NRC), Egypt.

\section{Preparation of MGE and NPE extracts}

Each dried powdered aerial parts (500 mg) of MG and NP was, separately, extracted by cold percolation in ethanol for 24 hrs. Each extract was, separately, recovered and ethanol was further added to the plant material, and the extraction was continued. The process was repeated 3 times and pooled together. The extracts were, separately, concentrated under reduced pressure $(22-26 \mathrm{mmHg})$ at $40^{\circ} \mathrm{C}$ to yield brown to greenish brown oily crude residue; 14.1 and $23.1 \mathrm{~g}$ of MGE and NPE, respectively. They were maintained in dark glass container, at $\left(-4^{\circ} \mathrm{C}\right)$ until use.

\section{Chemicals and apparatus}

Reference drugs atorvastatin and glibenclamide were purchased from Novartis Pharmaceuticals Chemical Company, Cairo, Egypt. Enzymelinked immunosorbent assay (ELISA) kits were provided by Invitrogen (U.S.A.) for IL-10, Eiaab (U.S.A.) for VCAM-1, ICAM-1, TNF- $\alpha$, CRP, and IL-10. All other chemicals and reagents were purchased from Biodiagnostic Company for diagnostic and research reagents, Cairo, Egypt. Rotary evaporator (Heidolph, Germany), electric grinder, centrifugation Eppendorf $\mathrm{S} 810 \mathrm{R}^{\circledR}$ (Germany), automatic biochemical analyzer Olympus AU400 ${ }^{\circledR}$ (USA), and Accu-chek active ${ }^{\circledR}$ blood glucose meter device with its stripes (Germany) were used.

\section{Experimental design}

\section{Animals}

A total of 70 adult male Wister rats (16-20 weeks age days; $150 \pm 10 \mathrm{~g}$ ) were selected for this study. They were provided by the Animal House of the NRC, Cairo, Egypt. All animals were kept in a controlled environment of air and temperature $\left(26-29^{\circ} \mathrm{C}\right)$, with a fixed light/ dark cycle for 2 weeks to acclimatize. They were allowed free access to water and diet ad libitum. This study had been approved by the Ethical Committee of the NRC, Egypt, which provided that the animals will not suffer at any stage of the experiment. The rats were randomly divided into seven groups of 10 animals each.

\section{Induction of hypercholesterolemia}

Hypercholesterolemia was induced in rats by feeding high fat/high cholesterol diet. Cholesterol was orally administrated at a dose of $30 \mathrm{mg} / 0.3 \mathrm{ml}$ olive oil/l $\mathrm{kg}$ animal 5 times a week for 12 consecutive weeks [13]. Lard fat was mixed with normal diet (1 $\mathrm{kg}$ of animal lard was added to $5 \mathrm{~kg}$ of normal diet). The hypercholesterolemia was determined by measuring the lipid profile including total cholesterol, low-density lipoprotein (LDL-c), high-density lipoprotein, and triglycerides. The HC rats were only used (data not shown).

\section{Routs of administration}

$\mathrm{HC}$ rats received an oral dose of $2 \mathrm{mg} / \mathrm{kg}$ body weight (BW) of plants extract dissolved in distilled water of the anti-HC reference drug; atorvastatin dissolved in distilled water orally by gastric intubation for 4 weeks.

\section{Induction of hyperglycemia}

Hyperglycemia was induced by intraperitoneal injection of a single dose of streptozocin (STZ, $45 \mathrm{mg} / \mathrm{kg}$ BW dissolved in $0.01 \mathrm{M}$ citrate buffer) immediately before use [5,13]. After injection, animals had free access for food, water and were given $5 \%$ glucose solution to drink overnight to encounter hypoglycemic shock. Animals were checked daily for the presence of glycosuria [14]. Animals were considered to be diabetic if glycosuria was present for 3 consecutive days. After 3 days of STZ injection fasting blood samples were obtained and blood sugar was determined ( $\geq 300 \mathrm{mg} / \mathrm{dL})$.

\section{Dose regimens and route of administration}

The rats were divided into the following groups; Group 1: Normal healthy control rats, Groups 2 and 3: Normal rats treated orally with $250 \mathrm{mg} / \mathrm{kg} \mathrm{BW}$ of crude extract of MGE and NPE, respectively, for 30 consecutive days. Group 4: Rats were considered as hyperglycemic (HG)-HC which classified as follows: Groups 5 and 6: HG-HC group rats oral administered $250 \mathrm{mg} / \mathrm{kg}$ BW crude ethanol extracts of MGE and NPE for 30 days, respectively. Group 7: HG-HC rats orally administered $2 \mathrm{mg} / \mathrm{kg} \mathrm{BW}$ of the anti-HC reference drug; atorvastatin [4,14] and the anti-HG glibenclamide reference drug at the dose of $10 \mathrm{mg} / \mathrm{kg}$ BW daily for 30 days $[13,14]$.

\section{Blood collection and tissue sampling}

By the end of the experiment (4 weeks), the animals of different groups were fasted for $12 \mathrm{hrs}$, weighted then blood samples were collected from the sublingual vein, then left to coagulate at room temperature and centrifuged at $3000 \mathrm{rpm}$ for 15 minutes. The clear, non-hemolyzed, sera were quickly removed and kept at $-80^{\circ} \mathrm{C}$ till used for biochemical investigations, kidney function parameters, inflammatory markers, and CAMs. Then, animals sacrificed using diethyl ether anesthesia. Kidney and heart tissue were rapidly excised and accurately weighed; for subsequent histopathological investigation.

\section{Biochemical examination \\ CAMs}

Rat soluble VCAM-1 concentration and ICAM-1 were determined using ELISA.

\section{Atherogenic markers}

In vivo quantitative measurements of IL-10, were performed by ELISA; a sandwich enzyme Immunoassay.

\section{Inflammatory markers}

In vivo quantitative measurements of the inflammatory mediator factors, tumor necrosis factor- $\alpha$ (TNF- $\alpha$ ), and CRP were estimated using diagnostic ELISA kits.

\section{The histological study}

At the end of the study, the animals were sacrificed, and their kidneys and hearts were removed, then small slices of them were fixed in $10 \%$ formalin solutions and processed routinely. Sections of $5 \mu \mathrm{L}$ thickness were cut and stained by hematoxylin and eosin ( $\mathrm{H}$ and $\mathrm{E}$ ) for histological examination.

\section{Image recognition subsystem (IRS) for rats' images}

We can describe the components of image recognition and classification subsystem (IRS) [15] and the process was carried out using the following illustration (Fig. 1).

\section{IRS input data}

The main dataset contains 48 images of rat' organs (kidney and heart) magnified 200 times used for evaluating IRS. The photomicrograph images were captured by Egyptian NRC experts. 


\section{IRS subsystem development}

In developing IRS, the current study passed through main steps of image recognition: Segmentation, feature extraction, and classification using digital image processing program. This procedure and the image analysis were accomplished using ImageJ v1.43u [16]. The program showed the following:

1. Preprocessing and segmentation of the images: In which, we enhance image quality and transform images in a proper format to deal with by image processing program.

2. Feature extraction and measurement: In which, the study discovered the most proper features and characteristics of the images

3. Image classification: In which, the study combined the results of the prior measurements. The object belongs to a class of images, according to certain appurtenance mathematical criteria.

\section{RESULTS}

The effect of MGE and NPE on endothelial dysfunction (CAMs)

Table 1 showed the effect of MGE and NPE extracts on adhesion molecules in HG associated with HC module (HG-HC group) and in different therapeutic groups. The present results demonstrate a significant decrease in ICAM-1 level with MGE and NPE supplemented to normal rats as compared to untreated control by 17.80 and $12.00 \%$, while insignificant change was detected in VCAM-1 level. HG-HC rats showed a significant increase in both VCAM-1 and ICAM- 1 levels by 80.43 and $40.27 \%$, respectively. ICAM-1 level was significantly decreased in HGHC treated rats with MGE, NPE, and drugs as compared to control, by percentages of improvement of 68.91, 29.93, and 33.22\%, respectively. VCAM-1 showed an insignificant decrease with MGE, NPE, and with amelioration percentages $31.25,24.30$, and $27.43 \%$, respectively. From results in Table 1, the highest amelioration percentage in ICAM-1 and VCAM-1 levels was obtained with MGE, followed by drugs and finally NPE.

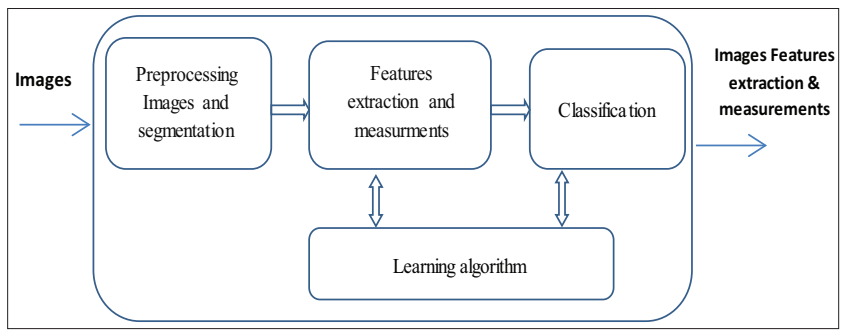

Fig. 1: Image recognition and classification process diagram
The anti-inflammatory effect of MGE and NPE

Table 2 indicated that, insignificant change in inflammatory cytokines markers; IL-10, CRP, and TNF- $\alpha$ in normal rat supplemented with MGE and NPE as compared to normal untreated control. HG-HC showed a significant increase in CRP and TNF- $\alpha$ by 85.93 and $50.99 \%$, respectively. While a significant decrease in IL-10 was noticed in HCHG with percentage decrease reached to $49.86 \%$ as compared to normal control rat was observed. Treatment of HG-HC rats with MGE provided insignificant change in IL-10 and TNF- $\alpha$ level while significant increases in CRP level as compared to normal control with percentage of amelioration 62.93. Furthermore, treatment with NPE showed the same effect of MGE as it showed insignificant change in IL-10 and TNF- $\alpha$ levels, while significant increase in CRP level with amelioration percentage 75.36\%. In addition, treatment of HG-HC rats with hypoglycemic glibenclamide and anti-HC atorvastatin showed the same results of MGE and NPE treatments as they declared insignificant change in both IL-10 and TNF- $\alpha$ as compared to normal control rats, while, significant increase in CRP level with percentage of improvement 52.49\%.

\section{The effect of MGE and NPE on kidney functions}

It is noticeable from Table 3 that there is no significant difference in the kidney function indices in control rats treated with each of the extract comparing to untreated normal one. With respect to the present results, HG-HC rats demonstrated marked increase in kidney function biomarkers with percentage 102.22 and $177.41 \%$, respectively, for urea and creatinine. Urea levels were returned to a normal value, showed insignificant change in HG-HC treated groups with MGE, NPE as well as standard drugs. While creatinine levels showed insignificant change with NPE and reference drugs while significant increase (63.22\%) with MGE with amelioration percentage of 114.19 (Table 3).

\section{Histopathological investigations of the kidney}

In Fig. 2a photomicrograph of control kidney section $\times 200$ showed renal cortex of renal corpuscle with normal glomerulus. The kidney tissue section of control group treated with S. tomentosa (Fig. 2b) showed no change in the kidney cells. Fig. 3 showed a photomicrograph of control kidney section treated with I. tricolor. Fig. 3a showed no change in normal cells structure of kidney cells. While section of $\mathrm{HC}+\mathrm{HG}$ group (Fig. 3b) showed a destructed epithelial lining of distal convoluted tubules stained with $(\mathrm{H}$ and $\mathrm{E}) \times 400$. Fig. 4 showed another photomicrograph of kidney tissue section of $\mathrm{HC}+\mathrm{HG}$ group (Fig. 4a) revealed proximal convoluted tubules and destructed epithelial lining $\times 400$. The kidney tissue section of $\mathrm{HC}+\mathrm{HG}$ group treated with S. tomentosa (Fig. 4b), showed normal pattern of proximal convoluted. Fig. 5 is the photomicrograph of $\mathrm{HC}+\mathrm{HG}$ group kidney

Table 1: Comparative effects of MGE and the NPE supplementation on adhesion molecules, ICAM-1 and VCAM-1 in different therapeutic groups

\begin{tabular}{|c|c|c|c|}
\hline Groups & Parameters & ICAM-1 & VCAM-1 \\
\hline Negative control & Mean \pm SD & $6.08 \pm 0.23 \mathrm{a}$ & $2.88 \pm 0.24 \mathrm{a}$ \\
\hline \multirow[t]{2}{*}{ Negative treated with MGE } & Mean \pm SD & $5.18 \pm 0.25 b$ & $2.43 \pm 0.41 \mathrm{ab}$ \\
\hline & $\%$ Change to control & 17.80 & 15.625 \\
\hline \multirow[t]{2}{*}{ Negative treated with NPE } & Mean \pm SD & $5.35 \pm 0.33 b$ & $2.85 \pm 0.23 a$ \\
\hline & $\%$ Change to control & 12.00 & 1.04 \\
\hline \multirow[t]{2}{*}{$\mathrm{HG}-\mathrm{HC}$} & Mean \pm SD & $10.97 \pm 0.37 \mathrm{c}$ & $4.72 \pm 0.17 \mathrm{c}$ \\
\hline & $\%$ Change to control & 80.43 & 40.27 \\
\hline \multirow{2}{*}{ HG-HC treated with MGE } & $\%$ Change to control & 21.38 & 9.027 \\
\hline & $\%$ of improvement & 68.91 & 31.25 \\
\hline \multirow[t]{3}{*}{ HG-HC treated with NPE } & Mean \pm SD & $7.09 \pm 0.28 b$ & $3.42 \pm 0.34 \mathrm{ab}$ \\
\hline & $\%$ Change to control & 21.21 & 15.97 \\
\hline & $\%$ of improvement & 29.93 & 24.30 \\
\hline \multirow[t]{2}{*}{ HG-HC treated with glibenclamide and atorvastatin } & Mean $\pm S D$ & $5.99 \pm 0.46 b$ & $2.51 \pm 0.095 \mathrm{ab}$ \\
\hline & $\%$ Change to control & 17.92 & 12.84 \\
\hline
\end{tabular}

Adhesion molecules are expressed in $\eta \mathrm{g} / \mathrm{ml}$. Data presented as mean \pm SD, $\mathrm{n}=10$. Statistical analysis is performed using costate and SPSS computer programs (Version 8). Unshared letters (a, b, and c) are significant at $\mathrm{p} \leq 0.05$. SD: Standard deviation, HG-HC: Hyperglycemia-hypercholesterolemia, MGE: Morning glory extract, NPE: Necklace pod extract, ICAM-1: Intercellular adhesion molecule-1, VCAM-1: Vascular cell adhesion molecule-1 
Table 2: Comparative effects of MGE and NPE supplementation on inflammatory markers in control and different therapeutic groups

\begin{tabular}{|c|c|c|c|c|}
\hline Groups & Parameters & IL-10 & CRP & TNF- $\alpha$ \\
\hline Negative control & Mean \pm SD & $87.52 \pm 1.73 \mathrm{~cd}$ & $29.69 \pm 1.24 \mathrm{e}$ & $49.24 \pm 0.33 b$ \\
\hline \multirow[t]{2}{*}{ Negative treated with MGE } & Mean \pm SD & $83.2 \pm 3.23 \mathrm{~cd}$ & $29.62 \pm 0.94 \mathrm{e}$ & $44 \pm 2.82 \mathrm{c}$ \\
\hline & $\%$ Change to control & 4.93 & 0.23 & 10.64 \\
\hline \multirow[t]{2}{*}{ Negative treated with NPE } & Mean \pm SD & $82.07 \pm 6.169 \mathrm{~d}$ & $30.0 .27 \pm 0.81 \mathrm{e}$ & $42.83 \pm 0.99 c$ \\
\hline & $\%$ Change to control & 6.22 & 1.135 & 1.04 \\
\hline \multirow[t]{2}{*}{$\mathrm{HG}-\mathrm{HC}$} & Mean \pm SD & $43.88 \pm 6.18 \mathrm{a}$ & $55.205 \pm 2.68 \mathrm{a}$ & $74.35 \pm 4.75 a$ \\
\hline & $\%$ Change to control & 49.86 & 85.93 & 50.99 \\
\hline \multirow{2}{*}{ HG-HC treated with MGE } & $\%$ Change to control & 9.23 & 23.00 & 7.209 \\
\hline & $\%$ of improvement & 36.24 & 62.93 & 43.78 \\
\hline \multirow[t]{3}{*}{ HG-HC treated with NPE } & Mean \pm SD & $74.17 \pm 4.33 c$ & $32.83 \pm 1.45 \mathrm{~d}$ & $51.75 \pm 3.59 b$ \\
\hline & $\%$ Change to control & 13.61 & 10.57 & 5.097 \\
\hline & $\%$ of improvement & 34.61 & 75.36 & 45.89 \\
\hline \multirow[t]{3}{*}{ HG-HC treated with glibenclamide and atorvastatin } & Mean \pm SD & $77.97 \pm 12.32 \mathrm{c}$ & $39.62 \pm 1.49 b$ & $53.83 \pm 3.75 b$ \\
\hline & $\%$ Change to control & 10.91 & 33.44 & 9.32 \\
\hline & $\%$ of improvement & 38.95 & 52.49 & 41.67 \\
\hline
\end{tabular}

CRP is expressed in $\eta \mathrm{g} / \mathrm{ml}$, TNF- $\alpha$ and IL-10 are expressed in $\rho \mathrm{g} / \mathrm{ml}$, Data presented as mean \pm SD, $\mathrm{n}=10$. Statistical analysis is performed using costate and SPSS computer programs (Version 7). Unshared letters (a, b, c, d, and e) are significant at p $\leq 0.05$. SD: Standard deviation, HG-HC: Hyperglycemia-hypercholesterolemia, MGE: Morning glory extract, NPE: Necklace pod extract, CRP: C-reactive protein, TNF- $\alpha$ : Tumor necrosis factor alpha, IL-10: Interleukin-10

Table 3: Comparative effects of MGE and NPE supplementation on kidney function in normal and different therapeutic groups

\begin{tabular}{|c|c|c|c|}
\hline Groups & Parameters & Urea & Creatinine \\
\hline Negative control & Mean \pm SD & $22.5 \pm 2.50 \mathrm{bc}$ & $0.155 \pm 0.013 \mathrm{c}$ \\
\hline \multirow[t]{2}{*}{ Negative treated with MGE } & Mean \pm SD & $23.5 \pm 2.19 c$ & $0.16 \pm 0.033 c$ \\
\hline & $\%$ Change to control & 4.44 & 3.22 \\
\hline \multirow[t]{2}{*}{ Negative treated with NPE } & Mean \pm SD & $21.00 \pm 2.83 b c$ & $0.14 \pm 0.021 \mathrm{c}$ \\
\hline & $\%$ Change to control & 6.66 & 9.67 \\
\hline \multirow[t]{2}{*}{$\mathrm{HG}-\mathrm{HC}$} & Mean $\pm S D$ & $45.50 \pm 3.81 \mathrm{a}$ & $0.43 \pm 0.021 \mathrm{a}$ \\
\hline & $\%$ Change to control & 102.22 & 177.41 \\
\hline \multirow{2}{*}{ HG-HC treated with MGE } & $\%$ Change to control & 15.55 & 63.22 \\
\hline & $\%$ of improvement & 86.66 & 114.19 \\
\hline \multirow[t]{3}{*}{ HG-HC treated with NPE } & Mean $\pm S D$ & $25.00 \pm 1.34 b$ & $0.17 \pm 0.008 c$ \\
\hline & $\%$ Change to control & 11.59 & 9.67 \\
\hline & $\%$ of improvement & 91.11 & 167.74 \\
\hline \multirow[t]{2}{*}{ HG-HC treated with glibenclamide and atorvastatin } & Mean \pm SD & $22.34 \pm 2.22 \mathrm{bc}$ & $0.17 \pm 0.018 \mathrm{c}$ \\
\hline & $\%$ Change to control & 0.711 & 9.67 \\
\hline
\end{tabular}

Creatinine and total urea levels are expressed in $\mathrm{mg} / \mathrm{dl}$. Data presented as mean $\pm \mathrm{SD}, \mathrm{n}=10$. Statistical analysis is performed using costate and SPSS computer programs (Version 7). Unshared letters (a, b, and c) are significant at $\mathrm{p} \leq 0.05$. SD: Standard deviation, HG-HC: Hyperglycemia-hypercholesterolemia, MGE: Morning glory extract, NPE: Necklace pod extract

section treated with I. tricolor (Fig. 5a), and it showed cells structure enhancement normal pattern of proximal convoluted. The kidney tissue section of $\mathrm{HC}+\mathrm{HG}$ group treated with atorvastatin and glibenclamide (Fig. 5b) revealed almost normal arranged of renal corpuscle with normal glomerulus stained with $(\mathrm{H}$ and $\mathrm{E}) \times 200$.

\section{Histopathological investigations of the heart}

Fig. 6a showed a histological organization of the heart of control rats. (Fig. 6b) Control rat treated with S. tomentosa showed no change in cardiac muscle. Fig. 7a was the histological section of the heart of control rats treated with I. tricolor. Fig. 7b was the heart tissue section of HC-HG rat, and it showed degenerative changes in heart muscle. Fig. 8a histological section of the heart of diabetic rats revealed disordered cardiac myofibrils (Fig. 8b). Heart tissue section of HC-HG group treated with S. tomentosa showed enhancement in cardiac muscle $(\mathrm{H}$ and $\mathrm{E}) \times 200$. Fig. $9 \mathrm{a}$ is the histological section of the heart of HC-HG rats treated with I. tricolor showing restore of most cardiac histology. (Fig. 9b) Heart tissue section of HC-HG group treated with atorvastatin and glibenclamide.

\section{DISCUSSION}

The present results show that, significant increase in ICAM and VCAM levels in HG-HC rats with percentages 80.43 and $40.27 \%$, respectively.

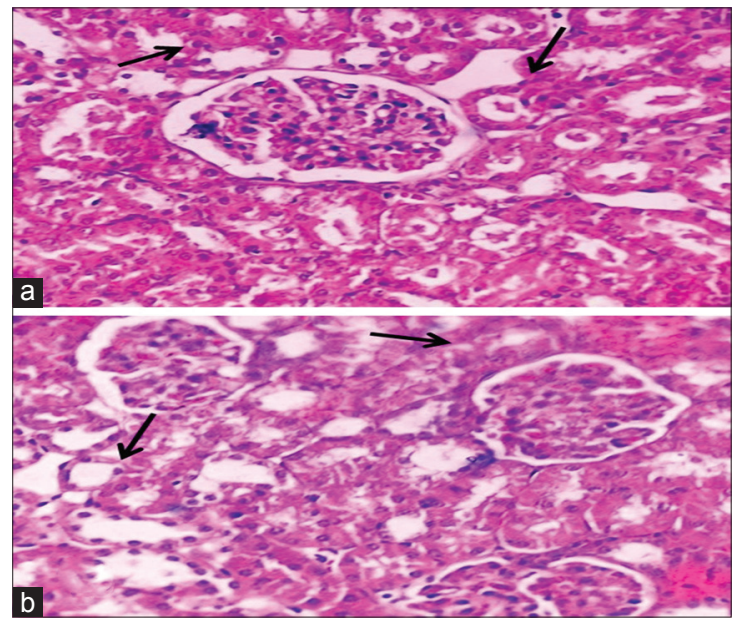

Fig. 2: (a) Photomicrograph of control kidney section $\times 200$ showed renal cortex of renal corpuscle with normal glomerulus.

(b) Kidney tissue section of control group treated with Sophora tomentosa showed no change in kidney cells, stained with (H and E) $\times 200$ 


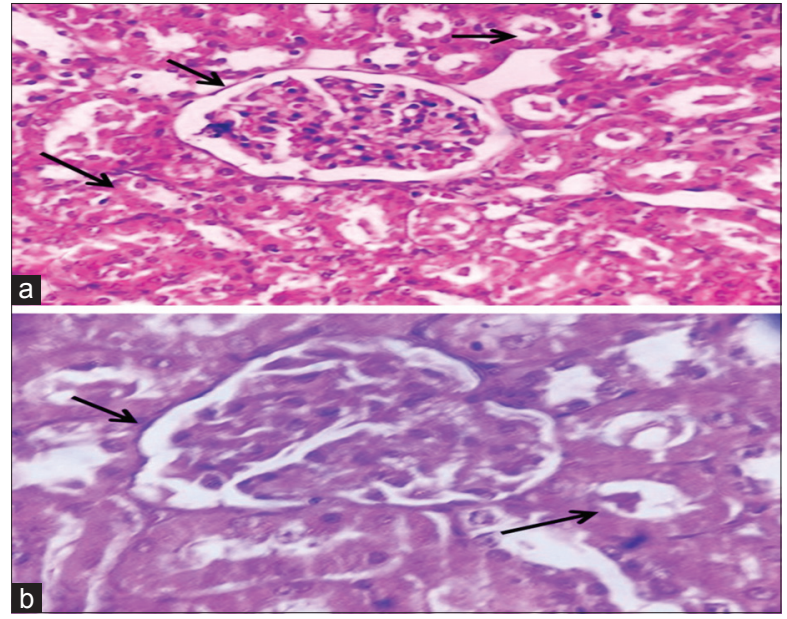

Fig. 3: (a) Photomicrograph of control kidney section treated with Ipomoea tricolor $\times \mathbf{2 0 0}$ showed no change in normal cells structure. (b) Kidney tissue section of hypercholesterolemia+hyperglycemia group showed destructed epithelial lining of distal convoluted tubules stained with $(\mathrm{H}$ and $\mathrm{E}) \times 400$

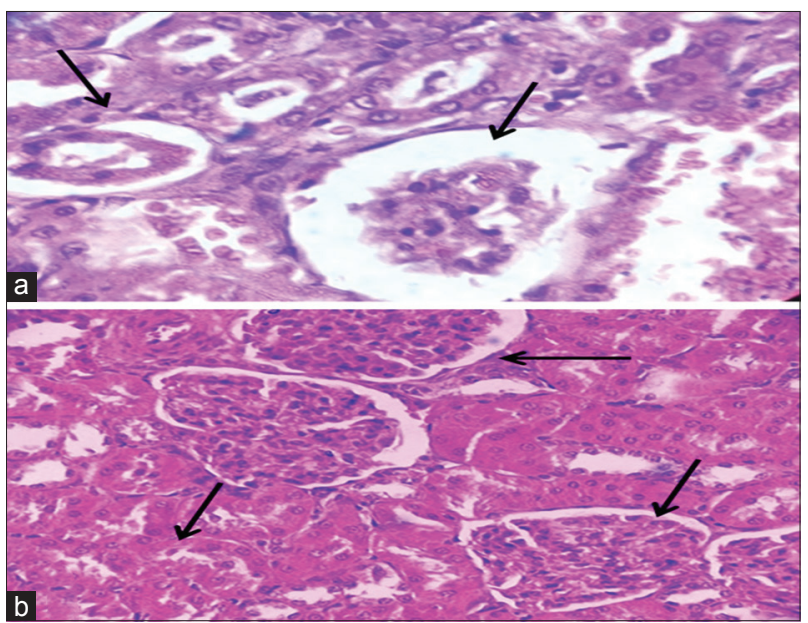

Fig. 4: (a) Another photomicrograph of kidney tissue section of hypercholesterolemia+hyperglycemia $(\mathrm{HC}+\mathrm{HG})$ group revealed proximal convoluted tubules show destructed epithelial lining $\times 400$. (b) Kidney tissue section of HC+HG group treated with Sophora tomentosa, showed normal pattern of proximal convoluted (arrow) stained with $(\mathrm{H}$ and $\mathrm{E}) \times \mathbf{2 0 0}$

While, a significant increase in ICAM level post MGE, NPE treatments as well as reference drugs with amelioration percentages 68.91, 29.93, and $33.22 \%$. While, VCAM level returned to a normal value, where it detected insignificant change post-treatment of HC-HG rats with MGE, NPE as well as reference drugs.

The cytokines can be represented in two shapes including the antiinflammatory markers such as IL- $1 \beta$, IL- 6 , and TNF- $\alpha$ and the second shape is the adhesion molecules such as ICAM-1 and VCAM-1 [17]. Large evidence has shown strong associations of circulating levels of endothelial adhesion molecules with insulin resistance in non-diabetic individuals or patients with Type 2 diabetes [17]. El-Baz et al. [2] supported the hypothesis that insulin stimulates nitric oxide output within the endothelial cells and hence it attenuates endothelial functions as adhesion molecules. It is well known that, diabetes mellitus enhance oxidative status and hence, oxidant stress promotes increased leukocyte adhesion to vascular endothelium [2]. Free radicals are well ascertained to stimulate, cytokine expression, division of cells, and at a high level, they can imitate damage of cell and subsequent death by

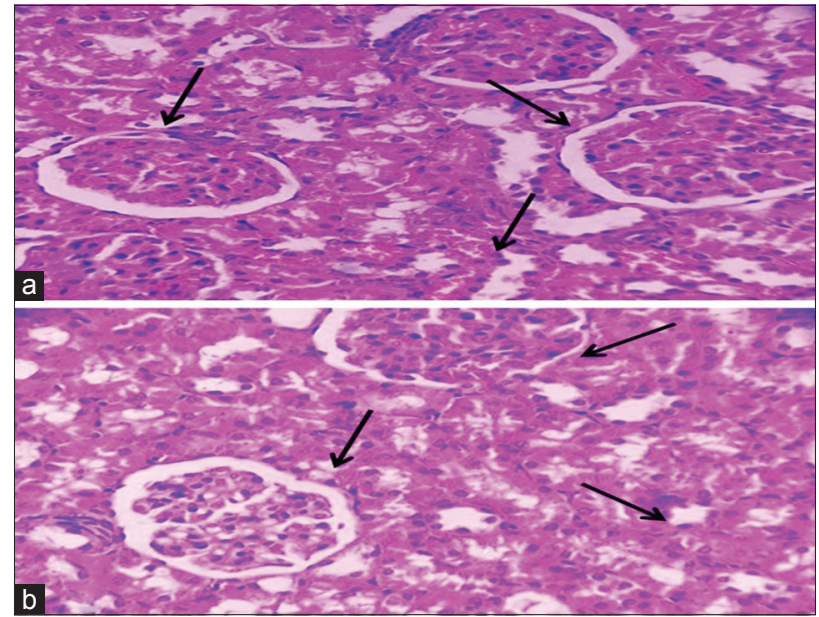

Fig. 5: (a) Photomicrograph of hypercholesterolemia + hyperglycemia $(\mathrm{HC}+\mathrm{HG})$ group kidney section treated with Ipomoea tricolor $\times 200$ showed cells structure enhancement normal pattern of proximal convoluted. (b) Kidney tissue section

of $\mathrm{HC}+\mathrm{HG}$ group treated with atorvastatin and glibenclamide revealed almost normal arranged of renal corpuscle with normal glomerulus stained with $(\mathrm{H}$ and $\mathrm{E}) \times \mathbf{2 0 0}$

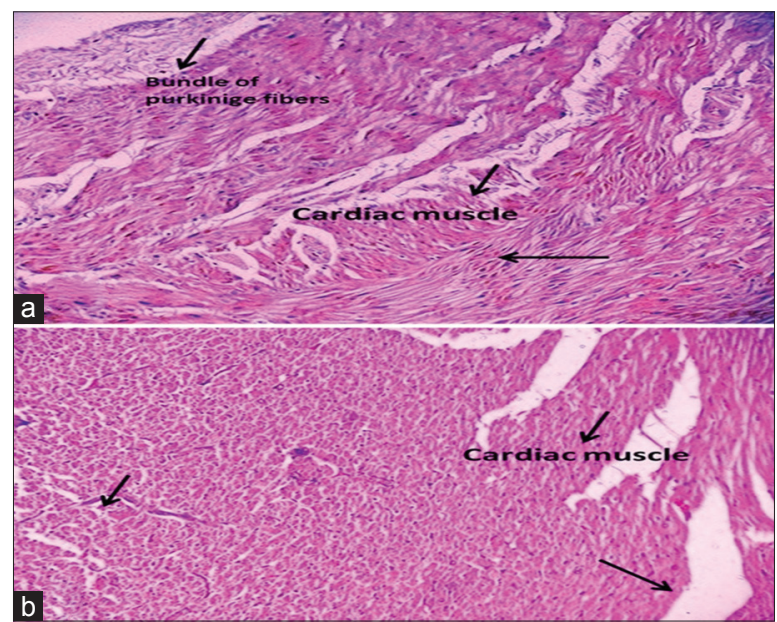

Fig. 6: (a) Histological organization of the heart of control rats $\times 200$. (b) Control rat treated with Sophora tomentosa showed no change in cardiac muscle, stained with $(\mathrm{H}$ and $\mathrm{E}) \times 200$

proteins, carbohydrates, lipid, and DNA oxidative modification [18]. This is combined with; oxidants lead to the activation of endothelial cells, VCAM-1, ICAM-1, E-selectin, and chemokines output [2].

In a good connection with the present results, the elevated levels of adhesion molecules in HG-HC condition may be explained on the basis of activation of macrophages that can stimulate several cytokines. It was induced the endothelial VCAM-1, the matter leading to macrophage accumulation and this consequently may lead to VCAM-1 expression $[2,8]$.

Each extract-treatment improved 1CAM-1 and VCAM-1 levels, as compared to normal control rats, whereas in comparison with diseased HC-HG rats, treatment with each separate extract as well as atorvastatin and glibenclamide significantly ameliorate both CAMs. On the basis of the presented data, both extracts were observed to inhibit the expression of VCAM-1/ICAM-1 and reduced VCAM-1 expression, as they considered as a protective agent against the progression of atherosclerosis. These effects of extracts may be due to antioxidative and anti-inflammatory effects that reduced the oxidation of LDL-c to ox-LDL-c $[5,18]$ 


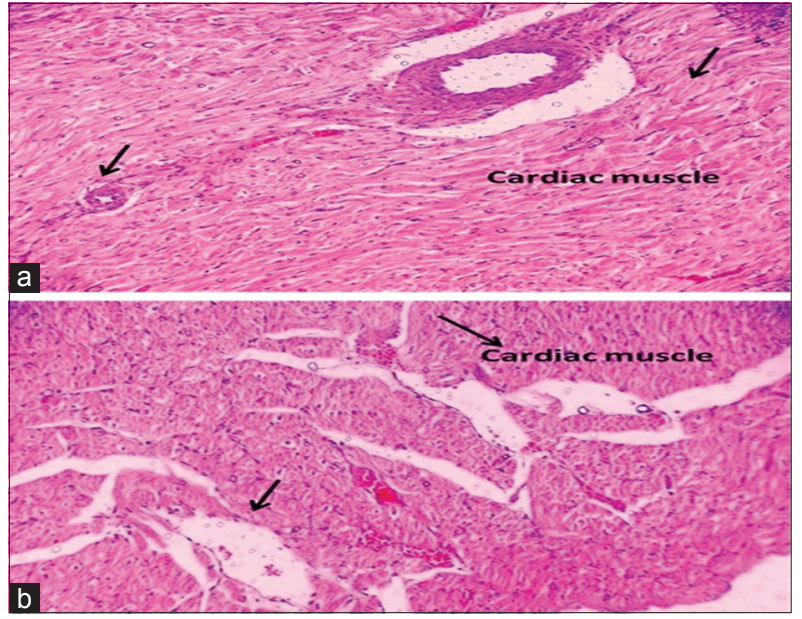

Fig. 7: (a) Histological section of the heart of control rats treated with Ipomoea tricolor. (b) Heart tissue section of hypercholesterolemia-hyperglycemia rat showing degenerative changes in heart muscle $\times 200(\mathrm{H}$ and $\mathrm{E})$

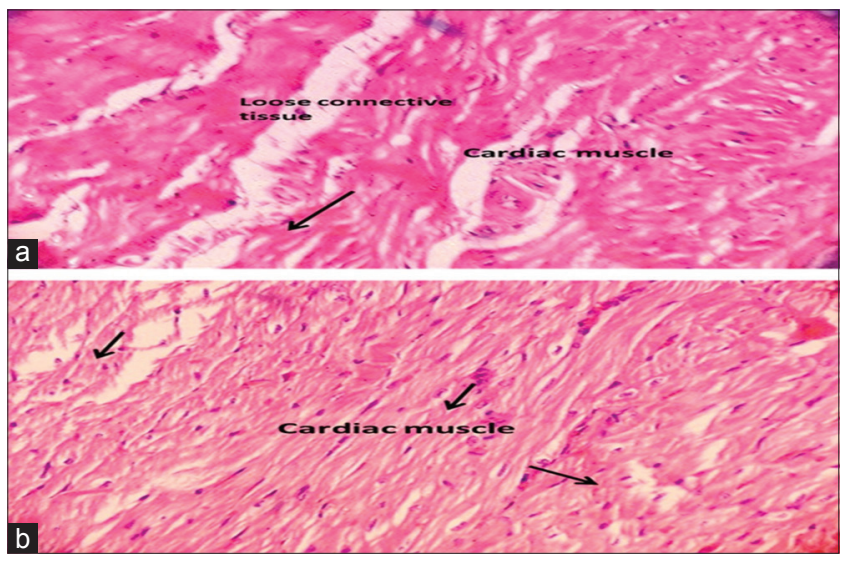

Fig. 8: (a) Histological section of the heart of diabetic rats revealed disordered cardiac myofibrils $\times 200$. (b) Heart tissue section of hypercholesterolemia-hyperglycemia group treated with Sophora tomentosa showed enhancement in cardiac muscle ( $\mathrm{H}$ and $\mathrm{E}) \times \mathbf{2 0 0}$
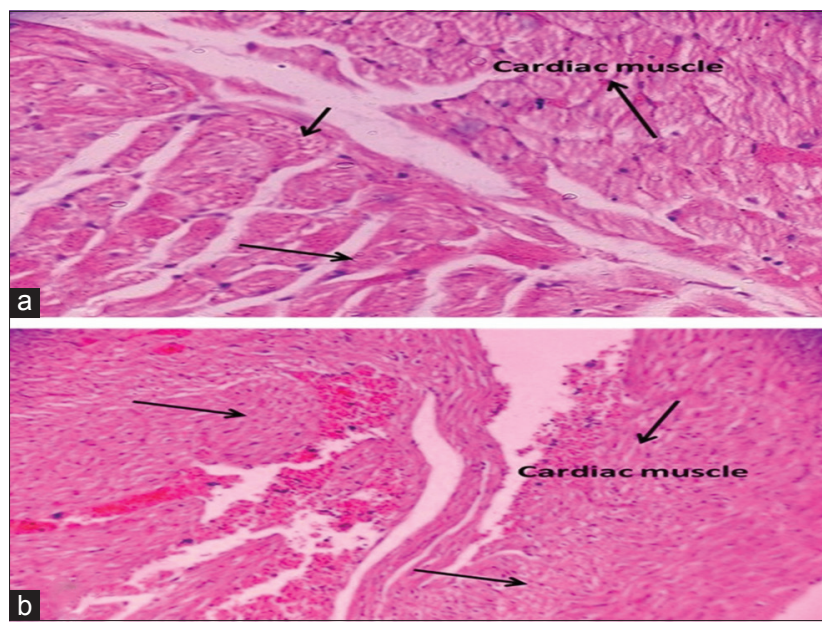

Fig. 9: (a and b) Histological section of the heart of hypercholesterolemia-hyperglycemia rats $\times 200$ treated with Ipomoea tricolor and showed restore of most cardiac histology
Hyperglycemia and ox-LDL-c all are resulting from a high level of free radicle as well as elevation in adhesion molecules secretion $[2,8]$. CAMs act as ligands on monocytes, eliciting adhesion of monocytes to endothelial cells, monocyte winding and transferred into subendothelial matrix. In diabetes, high glucose-stimulated cytokines of inflammation leading to dysfunction of endothelial cells by oxidative stress enhancement, raising inflammatory cells leakage and inducement of adhesion molecules on endothelial cells and monocytes [19].

Plant flavonoids and their derivatives are important constituents known as natural tender drugs. Compounds other than the bioactive flavonoids as alkaloids, coumarochromones, saponins, triterpene glycosides, phospholipids, polysaccharides, oligostilbenes, and fatty acids are reported in many Sophora species [6]

NP showed the presence of various structural features of flavonoids as isoprenylated flavonoids, isoflavonones, flavones, and flavonols [6]. These specific classes of compounds have wide bioactivity including anti-inflammatory, antioxidant, and suppression effect on adhesion molecules $[2,8,18]$. These compounds like many other antioxidant compounds have an inhibiting activity toward TNF- $\alpha$ stimulated VCAM-1 and ICAM-1 expression through a mechanism involving $\mathrm{NF}-\kappa \mathrm{B}$ and AP-1 [20]. Furthermore, these variable compounds may strengthen capillaries and other connective tissue and some function as anti-inflammatory and antioxidant agents. Hence, the endothelial dysfunction whether acute or chronic can be counteracted by the effect of these compounds $[6,20]$. Sophora radix has been applied for different disorders such as atherosclerosis and arrhythmias. Atherosclerosis is well established by hyperglycemia which acts as a critical point in diabetes complexity [21]. The present results clearly demonstrate amelioration with Sophora extract treatment to HG-HC rats. The plant extract showed suppressing effect on adhesion molecules and attenuation activity of VCAM-1 and ICAM-1 expression. Recently matrine, the main active ingredient of $S$. flavescens roots was reported to decrease adhesion molecules production in stimulated vascular smooth muscle cells [22]. In parallel with our study, it was found that, the extract of $\mathrm{S}$. tonkinensis root can inhibit the proliferation of melanoma cells, as well as their adhesion and movement [8]. In another study, a Sophora species (S. radix) extract showed inhibition of hyperglycemia-initiated up-regulation and production of VCAM- 1 on endothelial cell line through suppressing of NF- $\kappa \beta$ translocation [21]. These effects may be due to NPE active constituents as alkaloids and prenylated flavonoids $[6,22]$.

Genus Ipomoea has different secondary metabolites of variable activity [7]. Several phenolic compounds, namely, phenolic acids, flavonoids, and organic acids have been described in several Ipomoea species $[10,23]$. In addition, Ipomoea species are known for the presence of anthocyanins, which may also act as antioxidants $[10,20,23]$. It was reported that the isolated anthocyanins inhibit the progress of atherosclerotic injury and enhancements of oxidative status and vascular adhesion molecule- 1 in mice [23]. The leaves extract purple I. batatas showed inhibitive effect on cell adhesion and inflammatory mediation in endothelial cells of human aorta [24]. Hence; it is probable that the enhancement of endothelial dysfunction effect of MGE may be referred to its bioactive phenolic secondary metabolites as anthocyanins, flavonoids, and other flavonoids derivatives content [7].

Cytokines are classified as pro-inflammatory (TNF-a, IL$1,-6,-12,-15,-18$, and -32 ), beside, and anti-inflammatory cytokines; IL- 10 and TGF- $\beta$ elicited by stimulated macrophages, implicated in the control of inflammatory reactions and IFN- $\gamma$ and IL-4 from T-cells [25]. Anti-inflammatory cytokines such as IL-4, IL-10, IL-13, IFN- $\alpha$, and TGF- $\beta$ are involved in the downregulation of inflammation [25]. With respect to inflammatory markers CRP, TNF- $\alpha$ and IL-10 in control and different treated groups; CRP and TNF- $\alpha$ levels in hyperlipidemic diabetic rats recorded percentage increase reached to 85.93 and $50.99 \%$, respectively, while significant decrease in IL-10 (49.86\%) as compared to normal control rats. It was suggested that, inflammations 
have a crucial intermediary role in pathogenesis Type 2 diabetic, thereby inflammatory mechanisms can lead to joining diabetes with a number of commonly coexisting conditions $[1,17,24]$. TNF- $\alpha$ has been demonstrated as a marker of insulin resistance [2]. The proinflammatory mediators like TNF- $\alpha$ secretion play a key role in the elimination of invading microorganisms besides, the inhibition of TNF- $\alpha$ can improve insulin sensitivity in animals [2]. The present study shows that HC-HG rats showed strong activation of TNF- $\alpha$ and CRP, while suppression in IL-10 levels, as they all implicated in pathophysiological alterations that may accelerate inflammatory disease development in hypercholesterolemia and hyperglycemia [5]. This enhanced cytokines may induce polymorphonuclear leukocytes adhesion to endothelial surfaces, superoxide anion production, release of lysozyme, $\mathrm{H}_{2} \mathrm{O}_{2}$, and chemotaxis, suggesting their implication in increased inflammatory response [2]. High cholesterol and glucose levels connected with increased inflammatory biomarker CRP $[2,13]$. CRP is linked cardiovascular inflammation. Recently, in good agreement with the present finding Rada [26], found that CRP level was significantly elevated in HC-HG rats and this elevation is markedly linked with atherosclerosis in these animals. IL-10 is an immunoregulatory cytokines, elevated during inflammatory reaction to replace proinflammatory cytokine transmission. Hence, the output of some cytokines-related inflammation was susceptible to differ with illness degree [17]. In this study, the ameliorative levels of cytokines post-treatment with each extract may be related to the presence of flavonoid compounds as flavones, which has anti-inflammatory effect and significantly attenuated VCAM-1 and IL-8 expression [26].

Many Ipomoea species were reported as a good source of bioactive phenolic compounds, namely, phenolic acids, flavonoids, and anthocyanins [10]. These compounds may also act as antioxidant and anti-inflammatory agent $[7,10]$. Many other flavone derivatives were reported to inhibit COX-2 as well as nitric oxide synthase (NOS) [7,27]. Rutin, isolated from I. pes-caprae and I. fistulosa, decreases transfer of cells which was ascertained by the reduced levels of specific cytokines [7]. Furthermore, the aqueous extract and fractions of these plants neutralize inflammation induced by dangerous scorpion venom [27]. Anthocyanins and caffeoylquinic acid derivatives, isolated from purple I. batatas and I. cairica, have been reported to suppress histamine production in in vitro and decrease lipid peroxidation stimulated by $\mathrm{Fe}^{2+}$ and ascorbic acid in brain rat [7].

Inflammatory process is a critical cause of obesity. Many Ipomoea species, as I. involucrata and I. cairica are traditionally used in the treatment of toothache, rheumatic pains, and other inflammatory conditions [7].

Ipomoea constitutes many bioactive compounds which have different effects as antinociceptive and anti-inflammatory. The possible explanation for the antinociception is that these compounds reduced the release of pro-nociceptive mediators such as histamine [28]. In this study, the antiinflammatory activity and ameliorative level in adhesion molecules may be related to the presence of different bioactive compounds, viz, flavonols, flavones, chalcones, flavanones, isoflavones, isoflavonones, lavandulyl flavanones, and flavonol glycosides in the extract of MG (I. tricolor) [7]. Moreover, Krishna et al. [6] attributed the anti-inflammatory features of several active phytochemicals in Sophora species such as prenylated flavonoids and quinolizidine alkaloids to their abilities to moderate cell mitogen-activated protein kinases (MAPK) signaling pathways, redox balance. A prenylated flavonoid; sophoraflavanone $G$ and isoflavone glycoside; sophoricoside, trifolirhizin, a pterocarpan flavonoid isolated from many Sophora roots showed anti-inflammatory and inhibition of IL-6 and cyclooxygenase-2 in inflammatory response [29]. Several prenylated flavonoids are having a C-8 lavandulyl moiety were found to inhibit cyclooxygenase- 1 as well as 5 -lipoxygenase, and sophoraflavanone $\mathrm{G}$ was the most potent inhibitor against these eicosanoid generating enzymes among 19 prenylated flavonoids tested [30]. Sophoraflavanone G showed in vivo anti-inflammatory activity against mouse croton oil-induced ear edema and rat carrageenan paw edema via oral $(2-250 \mathrm{mg} / \mathrm{kg})$ or topical administration (10-250 $\mu \mathrm{g} /$ ear) [31]. Flavonostilbenes, alopecuroides $\mathrm{M}-\mathrm{O}$, were isolated from the root bark of $\mathrm{S}$. alopecuroides inhibit LPS-induced nitric oxide output in RAW 264.7 macrophages [21]. Arylbenzofurans and flavonoids isolated from the rhizomes and roots of S. tonkinensis showed IL-6 production inhibitory activity [32]. Antiinflammatory action of sophoraflavanone $\mathrm{G}$ isolated from $S$. flavescens in lipopolysaccharide-stimulated mouse macrophages [33]. It has an antiinflammatory property, reduced proinflammatory cytokines production via obstruction of the NF-KB and MAPK transmission. Production levels of interferon-gamma and TNF- $\alpha$ were decreased by the extract of root of S. flavescens (Sophora radix, SR) in vivo [34]. Finally, the release of histamine and $\beta$-hexosaminidase and migration were inhibited by the treatment with this extract. Sophocarpine, a tetracyclic quinolizidine alkaloid, isolated from $S$. alopecuroides showed anti-inflammatory effect [35] on carrageenan-induced rat hind paw edema. In this study, the anti-inflammatory activity of NPE might attribute to the lowering of iNOS and cyclooxygenase- 2 expressions exerted by the quinolizidine alkaloids content [36].

Prenylated flavonoids are represented the active components that exhibit beneficial effects on human health. The prenylated apigenin, quercetin, and kaempferol, anti-inflammatory properties may be mediated by suppressing the effect of NF- $\mathrm{KB}$ and AP-1 transcription [7].

In this study, administration of both extracts significantly attenuated the disturbances in these cytokines; the results of this study clearly shows that the mean levels of the TNF-a and CRP were significantly decreased, while IL-10 were markedly increased in MGE and NPE-treated HC-HG rats, this is probably due to decrease in proinflammatory biomarkers, suggesting mediation of endothelial activation and amelioration in endothelial function, as it suggested by Krishna et al., [6] this may lead to decrease in atherosclerosis development and reduction in local production of the cytokines by inflammatory cells that have accumulated. The current study investigated expressions of TNF- $\alpha$, CRP, and IL-10. These expressions were increased by STZ treatment and increased the expressions of those were decreased by extract, especially the NPE extract.

The effects of hypercholesterolemia-hyperglycemia on renal function were assessed through measuring serum total urea and creatinine; highfat diet intake caused significantly increase in levels of serum total urea and creatinine as compared to normal rats. The elevated levels of urea may be interpreted due to stimulated protein catabolism and activation of amino acid deamination for gluconeogenesis [3]. The increase in serum urea level in HC-HG rats indicated deterioration, in the normal renal function of the animal, as the mechanism of removing urea from the blood might have been affected. It may also be signs of injury at the glomerular and tubular levels of the kidney. It is well-known that many biochemical and histopathological findings ascertained renal injury in hypercholesterolemia-hyperglycemia condition [5]. With respect to the present results, HC-HG rats show a significant increase in total urea and creatinine levels with percentage 102.22 and $177.41 \%$, respectively. Total urea level showed insignificant change in hyperlipidemic diabetictreated groups with MGE, NPE as well as reference drugs as compared to normal control rats. While creatinine exhibited insignificant change in hyperlipidemic diabetic-treated groups with NPE and reference drugs showed similar improvement percentages (167.74\%). Although significant increase was detected in creatinine level with MGE with amelioration percentage of $114.19 \%$. Serum creatinine and urea levels are signs of glomerular filtration rate but plasma creatinine is more sensitive index of kidney function as compared to plasma urea level because of the achieving role of creatinine in most of the requirements for a perfect filtration marker [3]. The raised content of plasma creatinine and urea levels may be an indicator on the prerenal problem such as volume depletion and may be joined with the impaired function of the nephrons [3].

In this study, the higher percentages of amelioration in ICAM, VCAM and the proinflammatory IL-10 levels were recorded by MGE 
treatment. NPE showed higher percentages of amelioration in CRP, total urea and creatinine levels. The effect of many quinolizidine alkaloids, as oxymatrine isolated from different Sophora species, viz., S. tonkinensis and S. flavescens, markedly reduced blood glucose, serum creatinine, and blood urea nitrogen in diabetic rats and improved diabetes-induced glomerular and tubular architectures changes [37]. Moreover, plants contained significant levels of phenolic compounds have displayed high antioxidant and anti-inflammatory activities [38]. They markedly improved oxidative status and decreased glycation end products, transforming growth factor- $\beta 1$, and inflammatory cytokines in diabetic rats [17]. Another study suggested that $S$. flavescens has kidney protective potential from oxidative damage by the presence of many active compounds as; sophoraflavanone $\mathrm{G}$ and kurarinone and the radical generator 2,2'-azobis(2-amidinopropane) dihydrochloride in renal epithelial LLC-PK ${ }_{1}$ cells [6].

Genus Ipomoea is a good source of many bioactive secondary metabolites [7]. I. batatas (purple sweet potato) showed significant reduction of the expression level of kidney nucleotide-binding domain and leucine-rich repeat pyrin 3 domain (NLRP3) and Caspase-1, resulted in decline of IL-1 $\beta$. In addition, I. batatas suppressed the I kappa $\beta$ kinase $\beta$ (IKK $\beta$ ) stimulation and the nuclear translocation of NF-KB [39].

Histopathological investigation of the kidney and the heart supported by their IRS could conclude that both IRS and histopathological changes in kidney section as focal necrosis, tubular epithelial necrosis, tubular dilation, and vascular wall thickening seen in the $\mathrm{HC}+\mathrm{HG}$ group regressed with $S$. tomentosa or I. tricolor treatment, revealed almost normal arranged of renal corpuscle with normal glomerulus and might prevent the development of diabetic nephropathy. $\mathrm{HH}+\mathrm{HG}$ group treated with atorvastatin and glibenclamide, showing almost normal arranged kidney cell.

The analysis of statistical results has revealed that rats in the HC+HG group showed functional alteration of the myocardial microcirculation that may explain the left ventricular systolic dysfunction. Finally, the treatment by $S$. tomentosa or I. tricolor showed the decrease of myocardial perfusion reserve during the treatment, also reducing the degenerative changes in the myocardium, and restoring nuclei shape of the cardiomyocyte.

\section{CONCLUSION}

These results demonstrated that two plants extracts may be a candidate intelligent anti-inflammatory nutraceuticals and these extracts could be applied effectively to reduce renal complications in parallel to hypercholesterolemia associated diabetes. An image recognition system was useful to investigate renal injury images.

\section{ACKNOWLEDGMENT}

The authors wish to thank the financial support from National Research Centre (NRC) at Egypt for funding and supporting this research through the internal research project.

\section{REFERENCES}

1. Pickup JC. Inflammation and activated innate immunity in the pathogenesis of Type 2 diabetes. Diabetes Care 2004;27(3):813-23.

2. El-Baz FK, Aly HF, Khalil WK, Booles HF, Saad SA. Jatropha curcas repairing effect on adhesion molecules, DNA damage and gene expression alteration in STZ-induced diabetic rats. Int J Pharm Bio Sci 2015;6(3):198-214.

3. Aly HF, Abd-Alla HI, Ali SA, Aba-Alez R, Abu-Krisha MT, Mamdouh MM. Statistical data analysis which result from the biodiagnosis and bio-treatment of injured rats with the hyperlipidemia and hyperglycemia diseases. Asian J Pharm Clin Res 2016;9(3):122-30.

4. Abu-Gabal NS, Abd-Alla HI, Mohamed NZ, Aly HF, Shalaby NM. Phenolics composition, hypolipidemic, hypoglycemic and antioxidative effects of the leaves of Fortunella japonica (Thunb.) Swingle. Int J Pharm Pharm Sci 2015;7:55-63.
5. Mohamed NZ, Abd-Alla HI, Aly HF, Mantawy M, Ibrahim N, Hassan S. CCl4-induced hepatonephrotoxicity: Protective effect of nutraceuticals on inflammatory factors and anti-oxidative status in rat. J Appl Pharm Sci 2014;4:87-100

6. Krishna PM, Rao KN, Sandhya S, Banji D. A review on phytochemical, ethnomedical and pharmacological studies on genus Sophora, Fabaceae. Rev Bras Farm 2012;22(5):1145-54

7. Meira M, da Silva EP, David JM, David JP. Review of the genus Ipomoea: Traditional uses, chemistry and biological activities. Rev Bras Farmacogn 2012;22. Available from: http://www.scielo.br/scielo. php?script=sci_arttext\&pid=S0102-695X2012000300031.

8. Huang $\mathrm{M}$, Deng S, Han Q, Zhao P, Zhou Q, Zheng S, et al. Hypoglycemic activity and the potential mechanism of the flavonoid rich extract from Sophora tonkinensis Gagnep. In KK-Ay Mice. Front Pharmacol 2016:7:288

9. Zhang D, Liu S, Yao M, Liu X, Lai H. Effects of vietnamese sophora root on growth, adhesion, invasion and motility of melanoma cells. Afr J Tradit Complement Altern Med 2013;11(1):62-6.

10. Miyazaki K, Makino K, Iwadate E, Deguchi Y, Ishikawa F. Anthocyanins from purple sweet potato Ipomoea batatas cultivar Ayamurasaki suppress the development of atherosclerotic lesions and both enhancements of oxidative stress and soluble vascular cell adhesion molecule-1 in apolipoprotein E-deficient mice. J Agric Food Chem 2008;56:11485-92

11. Efford N. Digital Image Processing-A Practical Introduction Using Java. $1^{\text {st }}$ ed. USA: Pearson Education Limited, Addison Wesley; 2000.

12. Keedwell E, Narayanan A. Intelligent bioinformatics: The application of artificial intelligence techniques to bioinformatics problems. In: The Atrium. Southern Gate, Chichester, West Sussex, England: John Wiley \& Sons Ltd.; 2005

13. Shalaby NM, Abd-Alla HI, Aly HF, Albalawy MA, Shaker $\mathrm{KH}$, Bouajila J. Preliminary in vitro and in vivo evaluation of antidiabetic activity of Ducrosia anethifolia Boiss. and its linear furanocoumarins. Biomed Res Int 2014;2014:480545.

14. Abd-Alla HI, Albalawy MA, Aly HF, Shalaby NM, Shaker KH. Flavone composition and anti-hypercholesterolemic and anti-hyperglycemic activities of Chrysanthemum coronarium L. Z Naturforsch C 2014;69(56):199-208.

15. Bow ST. Pattern Recognition and Image Preprocessing. $2^{\text {nd }}$ ed. New York: Marcel Dekker Ltd.; 2002.

16. Rasband WS. ImageJ: Image Processing and Analysis in Java. USA: National Institutes of Health; 2012. Available from: http://www.rsbweb. nih.gov/ij/index.html.

17. Hwang SM, Kim JS, Lee YJ, Yoon JJ, Lee SM, Kang DG, et al. Antidiabetic atherosclerosis effect of Prunella vulgaris in $\mathrm{db} / \mathrm{db}$ mice with Type 2 diabetes. Am J Chin Med 2012;40(5):937-51.

18. Awad HM, Abd-Alla HI, Mahmoud KH, El-Toumy SA. In vitro antinitrosative, antioxidant, and cytotoxicity activities of plant flavonoids: A comparative study. Med Chem Res 2014;23:3298-307.

19. Venkatesan B, Valente AJ, Das NA, Carpenter AJ, Yoshida T, Delafontaine JL, et al. CIKS (Act1 or TRAF3IP2) mediates high glucoseinduced endothelial dysfunction. Cell Signal 2013;25(1):359-71.

20. Warner EF, Zhang Q, Raheem KS, O'Hagan D, O'Connell MA, Kay CD. Common phenolic metabolites of flavonoids, but not their unmetabolized precursors, reduce the secretion of vascular cellular adhesion molecules by human endothelial cells. J Nutr 2016;146(3):465-73.

21. Kwon KB, Kim EK, Lim JG, Shin BC, Song YS, Seo EA, et al. Sophorae radix extract inhibits high glucose-induced vascular cell adhesion molecule-1 up-regulation on endothelial cell line. Clin Chim Acta 2004;348(1-2):79-86.

22. Liu J, Zhang L, Ren Y, Gao Y, Kang L, Lu S. Matrine inhibits the expression of adhesion molecules in activated vascular smooth muscle cells. Mol Med Rep 2016;13(3):2313-9.

23. Grace MH, Yousef GG, Gustafson SJ, Truong VD, Yencho GC, Lila MA. Phytochemical changes in phenolics, anthocyanins, ascorbic acid, and carotenoids associated with sweetpotato storage and impacts on bioactive properties. Food Chem 2014;145:717-24.

24. Chao PY, Huang YP, Hsieh WB. Inhibitive effect of purple sweet potato leaf extract and its components on cell adhesion and inflammatory response in human aortic endothelial cells. Cell Adh Migr 2013;7(2):237-45.

25. Rada FH. Association of lipid fractions levels with cardiovascular disease. Asian J Pharm Clin Res 2017;10(3):180-2.

26. Sprague AH, Khalil RA. Inflammatory cytokines in vascular dysfunction and vascular disease. Biochem Pharmacol 2009;78(6):539-52.

27. Lima MC, Bitencourt MA, Furtado AA, Oliveira Rocha HA, 
Oliveira RM, da Silva-Júnior AA, et al. Ipomoea asarifolia neutralizes inflammation induced by Tityus serrulatus scorpion venom. J Ethnopharmacol 2014;153(3):890-5.

28. Ferreira AA, Amaral FA, Duarte ID, Oliveira PM, Alves RB, Silveira D, et al. Antinociceptive effect from Ipomoea cairica extract. J Ethnopharmacol 2006;105(1-2):148-53.

29. Zhou H, Lutterodt H, Cheng Z, Yu LL. Anti-inflammatory and antiproliferative activities of trifolirhizin, a flavonoid from Sophora flavescens roots. J Agric Food Chem 2009:57(11):4580-5.

30. Chi YS, Jong HG, Son KH, Chang HW, Kang SS, Kim HP. Effects of naturally occurring prenylated flavonoids on enzymes metabolizing arachidonic acid: Cyclooxygenases and lipoxygenases. Biochem Pharmacol 2001;62(9):1185-91.

31. Kim DW, Chi YS, Son KH, Chang HW, Kim JS, Kang SS, et al. Effects of sophoraflavanone G, a prenylated flavonoid from Sophora flavescens, on cyclooxygenase-2 and in vivo inflammatory response. Arch Pharm Res 2002;25(3):329-35.

32. Yoo H, Chae HS, Kim YM, Kang M, Ryu KH, Ahn HC, et al. Flavonoids and arylbenzofurans from the rhizomes and roots of Sophora tonkinensis with IL-6 production inhibitory activity. Bioorg Med Chem Lett 2014;24(24):5644-7.

33. Wun ZY, Lin CF, Huang WC, Huang YL, Xu PY, Chang WT, et al. Anti-inflammatory effect of sophoraflavanone G isolated from Sophora flavescens in lipopolysaccharide-stimulated mouse macrophages. Food Chem Toxicol 2013;62:255-61.

34. Choi BM, Oh GS, Lee JW, Mok JY, Kim DK, Jeong SI, et al. Prenylated chalcone from Sophora flavescens suppresses Th2 chemokine expression induced by cytokines via heme oxygenase-1 in human keratinocytes. Arch Pharm Res 2010;33(5):753-60

35. Song CY, Zeng X, Wang Y, Shi J, Qian H, Zhang Y, et al. Sophocarpine attenuates toll-like receptor 4 in steatotic hepatocytes to suppress pro-inflammatory cytokines synthesis. J Gastroenterol Hepatol 2015;30(2):405-12.

36. Elsherbiny NM, Al-Gayyar MM. The role of IL-18 in Type 1 diabetic nephropathy: The problem and future treatment. Cytokine 2016;81:15-22.

37. Wang SB, Jia JP. Oxymatrine attenuates diabetes-associated cognitive deficits in rats. Acta Pharmacol Sin 2014;35(3):331-8.

38. Taie HA, Abd-Alla HI, Ali SA, Aly HF. Chemical composition and biological activities of two Solanum tuberosum cultivars grown in Egypt. J Pharm Pharm Sci 2015;7(6):311-20.

39. Shan Q, Zheng Y, Lu J, Zhang Z, Wu D, Fan S, et al. Purple sweet potato color ameliorates kidney damage via inhibiting oxidative stress mediated NLRP3 inflammasome activation in high fat diet mice. Food Chem Toxicol 2014;69:339-46. 